\title{
Hippocampal testosterone relates to reference memory performance and synaptic plasticity in male rats
}

\author{
Kristina Schulz ${ }^{1 \dagger}$ and Volker Korz ${ }^{1,2+*}$ \\ 1 Leibniz Institute for Neurobiology, Magdeburg, Germany \\ Center of Behavioral Brain Sciences, Magdeburg, Germany
}

Edited by:

Nuno Sousa, University of Minho,

Portugal

Reviewed by:

Therese Jay, Institut National de la

Santé et de la Recherche Médicale U894, France

Cheryl A. Frye, University at

Albany-State University of New York,

USA

\section{${ }^{*}$ Correspondence:}

Volker Korz, Neuronal and Behavioral Plasticity Research Group, Institute of Biology, Otto von Guericke University Magdeburg, Leipziger Strasse 44, Building 91, 39120 Magdeburg, Germany.

e-mail:volker.korz@ovgu.de

\section{${ }^{+}$Present address:}

Kristina Schulz, Brain Research

Institute, University of Zurich,

Switzerland; Volker Korz, Otto von

Guericke University Magdeburg,

Germany.
Steroids are important neuromodulators influencing cognitive performance and synaptic plasticity. While the majority of literature concerns adrenal- and gonadectomized animals, very little is known about the "natural" endogenous release of hormones during learning. Therefore, we measured blood and brain (hippocampus, prefrontal cortex) testosterone, estradiol, and corticosterone concentrations of intact male rats undergoing a spatial learning paradigm which is known to reinforce hippocampal plasticity. We found significant modulations of all investigated hormones over the training course. Corticosterone and testosterone were correlated manifold with behavior, while estradiol expressed fewer correlations. In the recall session, testosterone was tightly coupled to reference memory (RM) performance, which is crucial for reinforcement of synaptic plasticity in the dentate gyrus. Intriguingly, prefrontal cortex and hippocampal levels related differentially to RM performance. Correlations of testosterone and corticosterone switched from unspecific activity to specific cognitive functions over training. Correspondingly, exogenous application of testosterone revealed different effects on synaptic and neuronal plasticity in trained versus untrained animals. While hippocampal long-term potentiation (LTP) of the field excitatory postsynaptic potential (fEPSP) was prolonged in untrained rats, both the fEPSP- and the population spike amplitude (PSA)-LTP was impaired in trained rats. Behavioral performance was unaffected, but correlations of hippocampal field potentials with behavior were decoupled in treated rats. The data provide important evidence that besides adrenal, also gonadal steroids play a mechanistic role in linking synaptic plasticity to cognitive performance.

Keywords: gonadal steroids, stress, endogenous, learning, dentate gyrus, LTP

\section{INTRODUCTION}

Considerable effects of steroidal hormones on cognitive performance and synaptic plasticity in vitro and in vivo have been described (McGaugh et al., 1975; McEwen et al., 1977; Joels and de Kloet, 1989; Pavlides et al., 1993; Sinopoli et al., 2006). However, because most studies use adreno- or gonadectomized animals with hormone replacement, little is known about regulations of endogenous gonadal and adrenal steroids, especially in the brain. The present study aimed at contributing to fill this gap by investigating functional relations of steroids to behavior, memory, and hippocampal synaptic plasticity during a spatial learning task in intact male rats.

We chose a spatial learning paradigm, which is known to affect plastic processes, i.e., reinforce long-term potentiation (LTP) in the dentate gyrus. Such behavioral LTP reinforcement is characterized by the transference of an electrically induced protein synthesisindependent early LTP, present for $8 \mathrm{~h}$, into a protein synthesisdependent late form, lasting for $24 \mathrm{~h}$ and more (Seidenbecher et al., 1997; Uzakov et al., 2005). It is hypothesized that synthesis of plasticity-related proteins is stimulated by concurrent learninginduced heterosynaptic activation and that processing of these proteins by the electrically stimulated synapses results in the prolonged potentiation.

There is reason to believe, that expression of plasticity-related proteins is also modulated by steroid hormones. Classic steroid receptors act as transcription factors, providing an orchestrated gene expression during behavioral demands by direct binding to the DNA and by interaction with other steroid transcription factors (McEwen et al., 1977; Diamond et al., 1990; Cato et al., 1992; Herman, 1993). Intriguingly, hippocampal testosterone and estradiol levels may be regulated independently from peripheral levels. Aromatase, the key enzyme for conversion of testosterone to estradiol, is expressed in hippocampal neurons of both genders (MacLusky et al., 1994; Wehrenberg et al., 2001), the effects of estradiol in males being largely unknown. Moreover, the potential of local production of some steroids, termed neurosteroids, has given rise to the idea that steroid actions may occur in a synaptocrine fashion (Corpéchot et al., 1981; Agís-Balboa et al., 2006; Mukai et al., 2006; Ishii et al., 2007). The hippocampus is equipped with the full enzymatic machinery to produce estradiol and thus also testosterone de novo (Hojo et al., 2004; Balthazart et al., 2006).

The described mechanisms are prompting the assumption that regulation of estradiol and testosterone concentrations in task relevant brain areas might be relevant for specific behavioral performances and memories. However, studies that address these questions in intact animals are still missing. Therefore, we followed testosterone, estradiol, and corticosterone concentrations over a spatial learning task in serum probes as well as in two task relevant brain regions, the hippocampus and the prefrontal cortex (PFC), in male rats. Both structures contain receptors of all steroids of interest and are known to be involved in the formation of spatial 
memories (Handa et al., 1997; Hajszan et al., 2007, 2008). In this first part of the study we identified testosterone as key player in the task, being strongly linked to behavioral parameters in the training session that is related to LTP reinforcement. In the second part, we studied the effects of testosterone application on behavior and on LTP in trained and untrained rats.

\section{MATERIALS AND METHODS SUBJECTS}

Male Wistar rats (strain Schönwalde; bred in the colony of the institute) were housed individually in standard cages $(40 \mathrm{~cm} \times 25 \mathrm{~cm} \times 18 \mathrm{~cm})$ under a $12 \mathrm{~h}$ light/dark regimen with lights on at $6 \mathrm{am}$. Animals had access to food (ssniff, R/M-H, Soest, Germany) and tap water ad libitum. All experiments were carried out in compliance with the national animal care guidelines and with permission of the regional council of Saxony-Anhalt, Germany.

\section{HOLEBOARD AND TRAINING PROTOCOL}

Spatial learning was assessed using a $1 \mathrm{~m} \times 1 \mathrm{~m}$ holeboard maze (COGITAT by Cognitron GmbH, Göttingen, Germany). Five out of 36 regularly arranged holes (diameter $6 \mathrm{~cm}$, depth $8 \mathrm{~cm}$ ) were baited (dustless precision pellets, $45 \mathrm{mg}$, Bioserv ${ }^{\circledR}$ ) over repeated trials, such that rats learned the pattern of baited holes (Figure 1A left panel). Photo sensors (detection system COGITAT) registered the rat's entries according to the depth as "inspection" (INS; upper beam), "visit" (VIS; middle light beam), and "removal of the pellet" (bottom light beam), respectively (Figure 1A right panel). The holeboard was kept in a soundproof, evenly lit room. The track of the animal was recorded (Field2020 for PLUS+, HVS Image Ltd., UK) and the time spent in areas surrounding the unbaited holes ("cells") and baited holes ("special cells") was calculated. Orienting landmarks on the arena enclosure (cue cards $30 \mathrm{~cm} \times 48 \mathrm{~cm}$ ) and outside the board (equipment) were clearly visible for the animal.

In order to motivate the rats to search for food pellets, they were mildly food-deprived (85\% of the initial body weight after food restriction to 6-7 g/day over 3 days). Animals were familiarized with the experimenter and the set-up before the training was performed on three consecutive days with each session starting at $9 \mathrm{am}$. Every trial lasted for $120 \mathrm{~s}$ or until all five pellets were eaten. It was differentiated between reference memory errors (RME; visits and inspections of non-baited holes) and working memory errors (WME; re-visits and re-inspections of initially baited holes of which the pellet had already been eaten). The board was wiped after each trial in order to spread odor cues. The interval between two successive trials was $20 \mathrm{~min}$.

\section{PART 1}

\section{Experimental groups for hormone assaying}

Animals were sacrificed $15 \mathrm{~min}$ or $24 \mathrm{~h}$ after a session creating five different training groups (see Figure 1B). Group $1(\mathrm{G} 1 n=8)$ and the trained control of group $2(\mathrm{tcG} 2 n=8)$ experienced 5 trials, group $2(\mathrm{G} 2 n=8)$ and the trained control of group 3 (tcG3 $n=8$ ) 9 trials and group 3 (G3 $n=12) 10$ trials. Food-deprived untrained animals (chamber controls) were sacrificed isochronously with each group ( $n=2-4$ per group). Non-food-deprived (non-fd) animals $(n=6)$ served as controls for the influence of food deprivation.

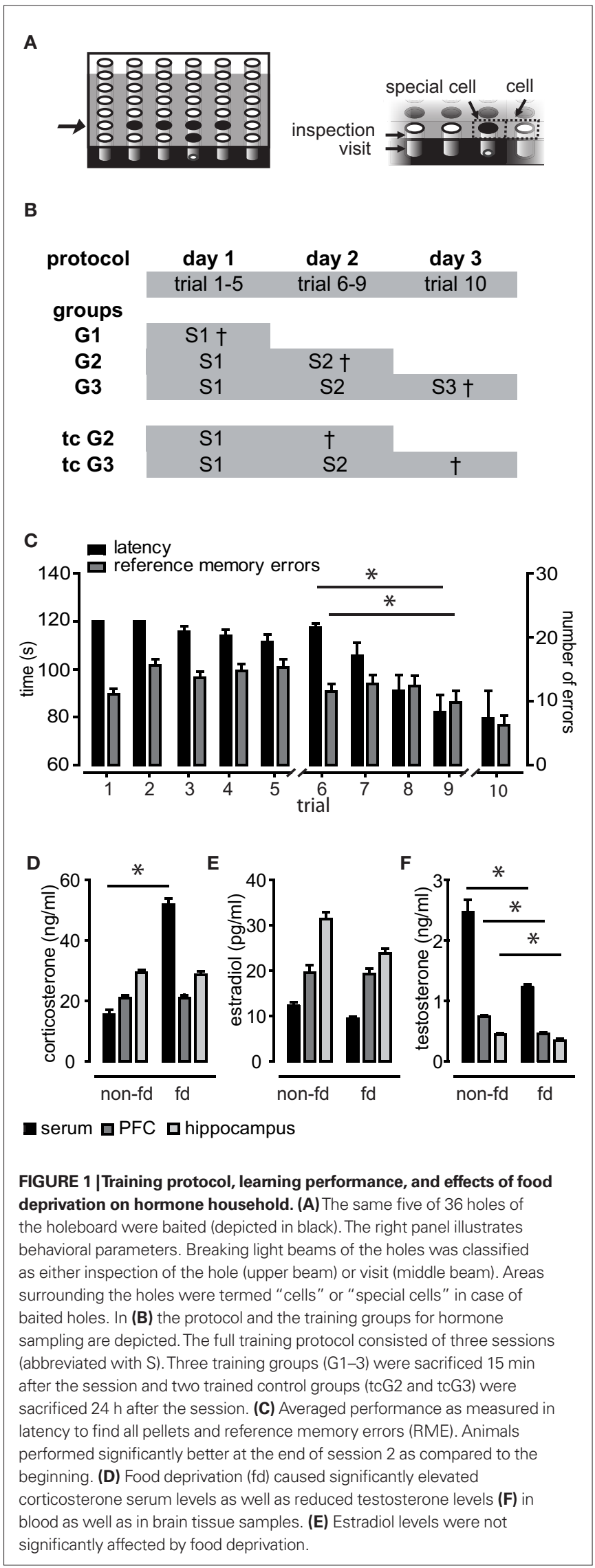




\section{Tissue sampling and hormone assaying}

Animals were decapitated and trunk blood was collected in vials containing clot activator (REF 41.1500.005, Sarstedt). The PFC and the right dorsal hippocampus were rapidly dissected, the tissue was homogenized (Biovortexer No. 1083; Biospec products) and diluent (Sample diluent, IBL Hamburg, REF KLZZ731) was added to reach a final volume of $100 \mu \mathrm{l} / \mathrm{mg}$ tissue weight. All samples were centrifuged ( $5 \mathrm{~min}, 10000 \mathrm{rpm}$ ); the supernatant was aliquotized and stored at $-20^{\circ} \mathrm{C}$. For hormone assaying enzyme-linked immunosorbent assay (ELISA) was performed. Briefly, probes were thawed and diluted (brain probes 1:3, 1:2 respective 1:2, serum probes $1: 10,1: 5$ respective $1: 10$ in testosterone, $17 \beta$-estradiol, and corticosterone assays). Samples and standards were applied in duplicate. OD values were measured at $450 \mathrm{~nm}$ in a micro plate reader (SpectraMax ${ }^{\circledR}$ Plus384). The type of assay used did not distinguish between the protein bound and the free hormone, which is an essential difference as it is assumed that only free hormone is active. For the time being, there was no ELISA available for measuring rat steroid binding proteins. All assays had little interference with other steroids, thus being highly specific. For the testosterone assay (Testosterone Saliva ELISA by IBL Hamburg) the limit of detection (LOD) was $2.0 \mathrm{pg} / \mathrm{ml}$ and intra-assay and inter-assay coefficients of variation were 8.2 and 5.5\%, respectively. The estradiol kit (17betaEstradiol Saliva ELISA by IBL Hamburg) had a LOD of $0.4 \mathrm{pg} / \mathrm{ml}$. Intra-assay and inter-assay coefficients of variation was maximally 9.9 and $11.1 \%$, respectively. In the corticosterone kit (AssayMax Corticosterone ELISA Kit Assaypro; catalog number EC3001-1) the minimum detectable dose of corticosterone was $40 \mathrm{pg} / \mathrm{ml}$ and intra-assay and inter-assay coefficients of variation were 5.0 and $7.0 \%$, respectively. Estradiol could not be detected in every probe. Values detected below the LOD were kept; probes with undetectable amounts of estradiol were set equal to 0 . Data for serum samples are given as weight $/ \mathrm{ml}$ and for brain samples as weight/g wet tissue weight (ww). Thus units are directly contrastable.

\section{PART 2}

\section{Electrode and cannula implantation}

Male rats ( 7 weeks, about $300 \mathrm{~g}$ ) were implanted a double recording electrode, a bipolar stimulation electrode, and a cannula for intracerebroventricular (icv) application of substances. Anesthesia was induced by ip application of $40 \mathrm{mg} / \mathrm{kg}$ Nembutal. Additional narcotic was applied when indicated. The head was stereotactically fixed (TSE System GmbH, Germany) such that bregma was $1 \mathrm{~mm}$ higher than lambda. The bipolar stimulation electrode was implanted in the right medial perforant path $(-6.9 \mathrm{~mm} \mathrm{AP}, 4.1 \mathrm{~mm}$ ML, 2.0-2.4 mm DV). The double recording electrode consisted of a pair of insulated stainless-steel wires ( $125 \mu \mathrm{m}$ in diameter) of which the inner tip was shortened by $400 \mu \mathrm{m}$. Stereotactic implantation into the right dentate gyrus (-2.8 $\mathrm{mm} \mathrm{AP,} 1.8 \mathrm{~mm} \mathrm{ML})$ yielded optimal signals at a depth of 2.8-3.4 $\mathrm{mm}$. The shorter tip was then situated in the laminar moleculare and recorded the slope $(\mathrm{mV} / \mathrm{ms})$ of the negative field excitatory postsynaptic potential (fEPSP); the longer tip reached into the granule cell layer and recorded the population spike amplitude (PSA), the difference in millivolt between the first positive and the first negative deflection of the signal. The cannula was implanted into the right lateral ventricle $(-0.4 \mathrm{~mm} \mathrm{AP,} 1.5 \mathrm{~mm} \mathrm{ML})$. Coordinates are based on the atlas of Paxinos and Watson (1998). Electrodes and sockets were fixed with dental cement. Animals were given at least 1 week to recover before the experiment. Positioning of the electrodes and the cannula was verified by histology of a random sample of brains. Cannula positioning was additionally verified after the experiment in each animal by application of angiotensin $(5 \mu \mathrm{g}$ solved in $\mathrm{NaCl}$ ), which induces drinking behavior when applied into the ventricular system.

\section{Electrophysiological recording protocols}

Animals were food-deprived prior to the experiment. A flexible cable connected the electrodes to a swivel, allowing for free movement of the animal. Biphasic constant current pulses $(0.1 \mathrm{~ms}$ per half wave) were delivered by stimulators (A-M Systems, Isolated Pulse Stimulator, Model 2100) which were triggered via pwin (PWIN, Institute of Neurophysiology, Magdeburg). Evoked field potentials were amplified (100×; differential amplifier, Inh; Science Products, Hochheim, Germany), sampled at a rate of $10 \mathrm{kHz}$, filtered (high pass: $0.1 \mathrm{~Hz}$; low pass: $5 \mathrm{kHz}$ ), and converted by an analog-to-digital interface (CED 1401+; Cambridge Electronic Design, Cambridge, UK) for storage on a PC. fEPSP and PSA were alternatively recorded every $15 \mathrm{~min}$ (mean of five stimuli with $10 \mathrm{~s}$ inter-stimulus interval). Stimulation intensities were adjusted according to an input/output (I/O) curve of the prior day to evoke $40 \%$ of the maximal PSA and $60 \%$ of the maximal fEPSP, respectively, but never exceeded $0.4 \mathrm{~mA}$. Substance application took place $30 \mathrm{~min}$ before the weak tetanus (three bursts of 15 stimuli, $200 \mathrm{~Hz}, 0.1 \mathrm{~ms}$ pulse duration, $10 \mathrm{~s}$ interburst-interval) which was given at the intensity used for PSA recording. The criterion for sufficient potentiation for the fEPSP and PSA values was an initial potentiation greater than 120 and $180 \%$, respectively, in one of the first two measurements.

\section{Testosterone application}

A flexible tube allowed free movement of the animals during application of $1 \mu \mathrm{g}$ testosterone propionate (3221760, Sigma-Aldrich) into the lateral ventricle. The application volume of $5 \mu \mathrm{l}(200 \mu \mathrm{g} / \mathrm{ml}$ testosterone in $40 \%$ propandiol) was applied at a rate of $1 \mu \mathrm{l} / \mathrm{min}$ using a Hamilton syringe (CR700-20). To estimate effects on the hormone status, controls (cannulated, food-deprived, non-trained) were sacrificed $30 \mathrm{~min}$ after the application corresponding to the time point of LTP induction. The procedure took place at a similar time of the day as decapitation of unprepared animals as well as application time in electrophysiological studies. Brain samples, but no trunk blood, was taken and assayed.

\section{Cognitive reinforcement protocol}

Animals underwent the training protocol described in Section "Holeboard and Training Protocol." In the morning before the last session, a $1 \mathrm{~h}$ baseline was recorded; substance application and tetanus took place as described for the early LTP experiment (see Electrophysiological Recording Protocols). A measurement of the fEPSP and PSA was taken before behavioral manipulation. Fifteen to 18 minutes after the tetanus, animals performed the 10th trial, which corresponded to a time between 9.30 and $10.30 \mathrm{am}$. After the training, the recording proceeded as described for the early LTP protocol. 


\section{Paired pulse experiment}

In untrained, food-deprived animals two pulses were paired with intervals of 10, 20, 50, 70, and $120 \mathrm{~ms}$ (5 min inter-pair interval) at a stimulation intensity evoking $40 \%$ of the maximal PSA. For each interval, the average of three paired pulses $(10 \mathrm{~s}$ inter-stimulus interval) was calculated. One pulse set was measured prior to application, and further measurements took place at 30, $90 \mathrm{~min}, 3,6,8$, and $24 \mathrm{~h}$ after application.

\section{DATA ANALYSIS}

\section{Hormone data}

Normal distribution was tested using Shapiro-Wilk test. MannWhitney $U$ test was used to compare chamber controls to trained animals, and trained controls to animals sacrificed $15 \mathrm{~min}$ post session.

\section{Behavioral data}

The three measures latency, RME, and WME were used to assess performance differences between training groups. For each session MANOVA with groups as between-subject factors and the averaged latency, RME, and WME as within-subject factors was conducted. Paired Student's $t$-test was performed to compare each of the three named measures at the beginning and end of session 2 (trial 5 versus trial 9).

Behavioral data was correlated with hormone levels using twotailed Pearson correlation. Data from the last trial of each session and averaged data over each session and over the entire training experience was analyzed. To evaluate the most predictive parameters, multiple linear regression with stepwise exclusion was performed. This execution was chosen in spite of multicollinearity of some factors. Excluded variables most likely carry indeed predictive information, but could not add significantly to the model due to intercorrelation with included parameters. However, it is thought to help identifying the main variance predictor to direct future research.

In the cognitive reinforcement study, to evaluate effects of the application, data from trial 10 and savings, which indicate improvement from trial 9 to trial 10, and, thereby, control for pre-manipulation performance differences, were compared between vehicle and testosterone applied animals using $t$-test. Electrophysiological data (hour values from 1- to 24-h post induction) were correlated with behavioral parameters in trial 10 by two-tailed bivariate Pearson correlations.

\section{Electrophysiological data}

Group comparisons were performed with repeated-measure ANOVA with all values post induction, respectively, values from 5 to $24 \mathrm{~h}$ (the protein synthesis-dependent late phase of LTP) in trained animals. Student's $t$-test was performed to evaluate significant differences between testosterone treated and control animals at the $24 \mathrm{~h}$ time point.

Paired pulse data was analyzed using ANOVA. To evaluate group differences over the time in values of the five different stimulus intervals, repeated measure ANOVA with the sample time as withinsubject factor, the interval-size as measure, and treatment versus control as between-subject factor was performed. In order to test early and late effects independently, for each time point groups were compared by MANOVA with the five intervals as the within-subject factor and groups (control versus treated) as between-subject factor. In order to investigate whether the values of a certain interval changed differentially over time, repeated measure ANOVA was performed for each interval with sampling time as within-subject factor and groups (control versus testosterone) as between-subject factor.

All data are given in mean \pm SEM if not stated otherwise. The level for statistical significance was set at $p<0.05$.

\section{RESULTS \\ PART 1}

Male rats were trained on a spatial holeboard learning task. Independent groups were sacrificed at different time points of the training procedure (Figure 1B) and concentrations of testosterone, estradiol, and corticosterone in blood, hippocampal and prefrontal brain tissue samples were measured.

\section{Animals acquired the task in the second session}

The animals were required to learn a spatial pattern of baited holes by orientation toward environmental cues over repeated trials (10 trials distributed over three sessions; Figure 1). Learning performance of training groups was assessed by the two parameters latency and RME, which reflect the long-term memory for the spatial location of the holes. Recall was significantly better at the end of session $2 \mathrm{com}$ pared to the beginning ( $\operatorname{RME} p=0.006$; latency $p<0.001)$, indicating that the animals acquired the task in this session (Figure 1C). This is in compliance with previous studies (Uzakov et al., 2005). To judge whether the performance was similar in different groups and ensure that differences in hormone levels (see below) were not caused by a global difference in performance, we performed MANOVA with RME, latency, and WME as within-subject factors. WME indicate short-term memory, which is thought to be trial specific. The five independent training groups (Figure 1B) did neither differ on a linear combination of latency, RME, and WME in the first session $(p>0.1)$ nor in the second $(p>0.1)$.

\section{Food deprivation did affect only serum corticosterone levels but not brain tissue concentrations}

Rats were mildly food deprived to motivate them to search for food pellets. As food deprivation is a chronic stressor, it was expected to cause elevated corticosterone levels, which may in turn act on gonadal hormone release. When comparing the pooled food-deprived chamber controls $(n=16)$ to non-deprived controls $(n=6)$, corticosterone levels were elevated significantly in serum samples only $(U=11.00, z=-2.728, p=0.005)$, but not in brain tissues $(p>0.1$; Figure 1D). As expected, testosterone levels were lower in deprived animals, an effect which was present in all investigated samples (serum $U=17, z=-2.285, p=0.021$, hippocampus $U=20.00, z=-2.064, p=0.40$, PFC $U=11.00, z=-2.728, p=0.005$; Figure 1F). Food deprivation had no significant effect on estradiol levels ( $p>0.1$; Figure 1E).

\section{Alterations of endogenous steroidal hormones during spatial learning on short and long time scales}

We raised a profile of endogenous hormone levels over training experience (Figure 2). In order to identify changes related to the training experience rather than other factors, such as daytime of 

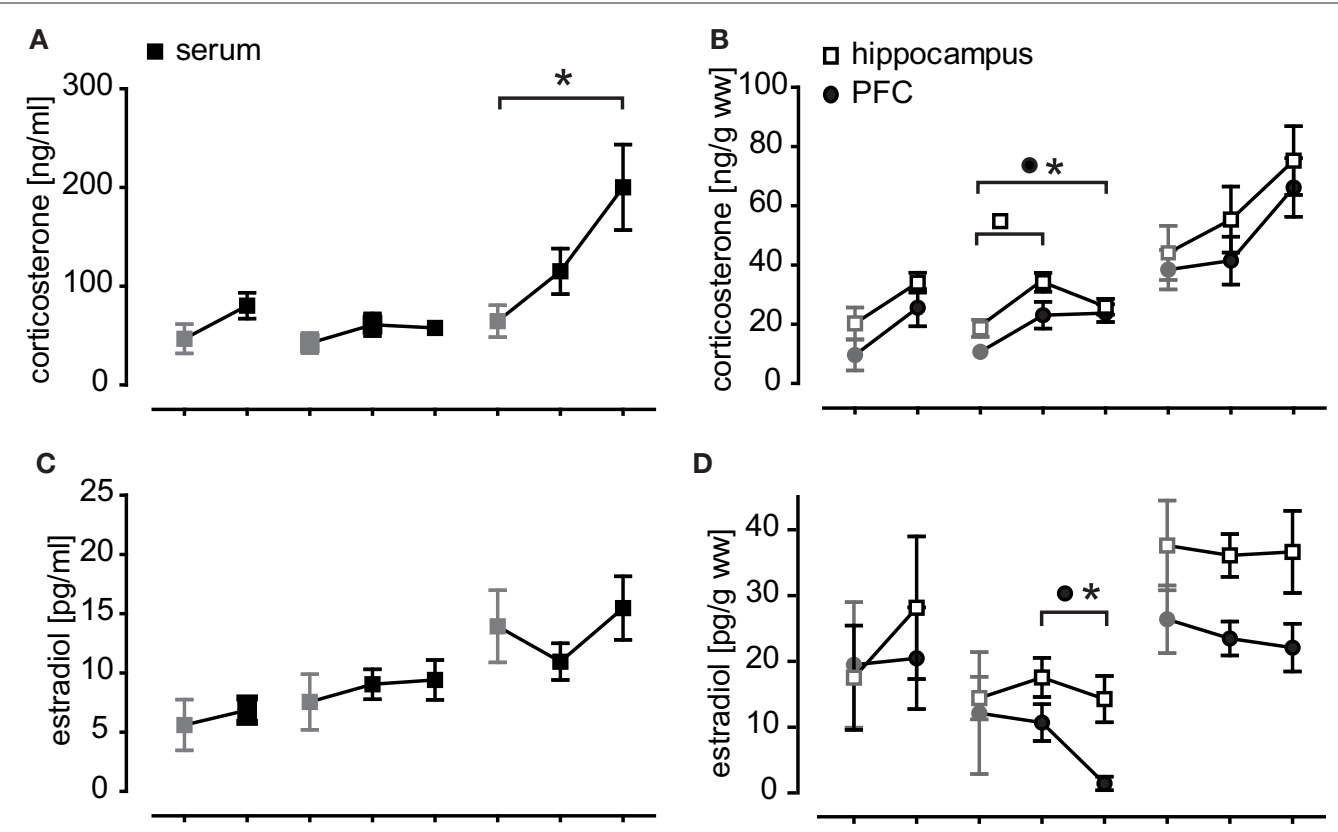

D
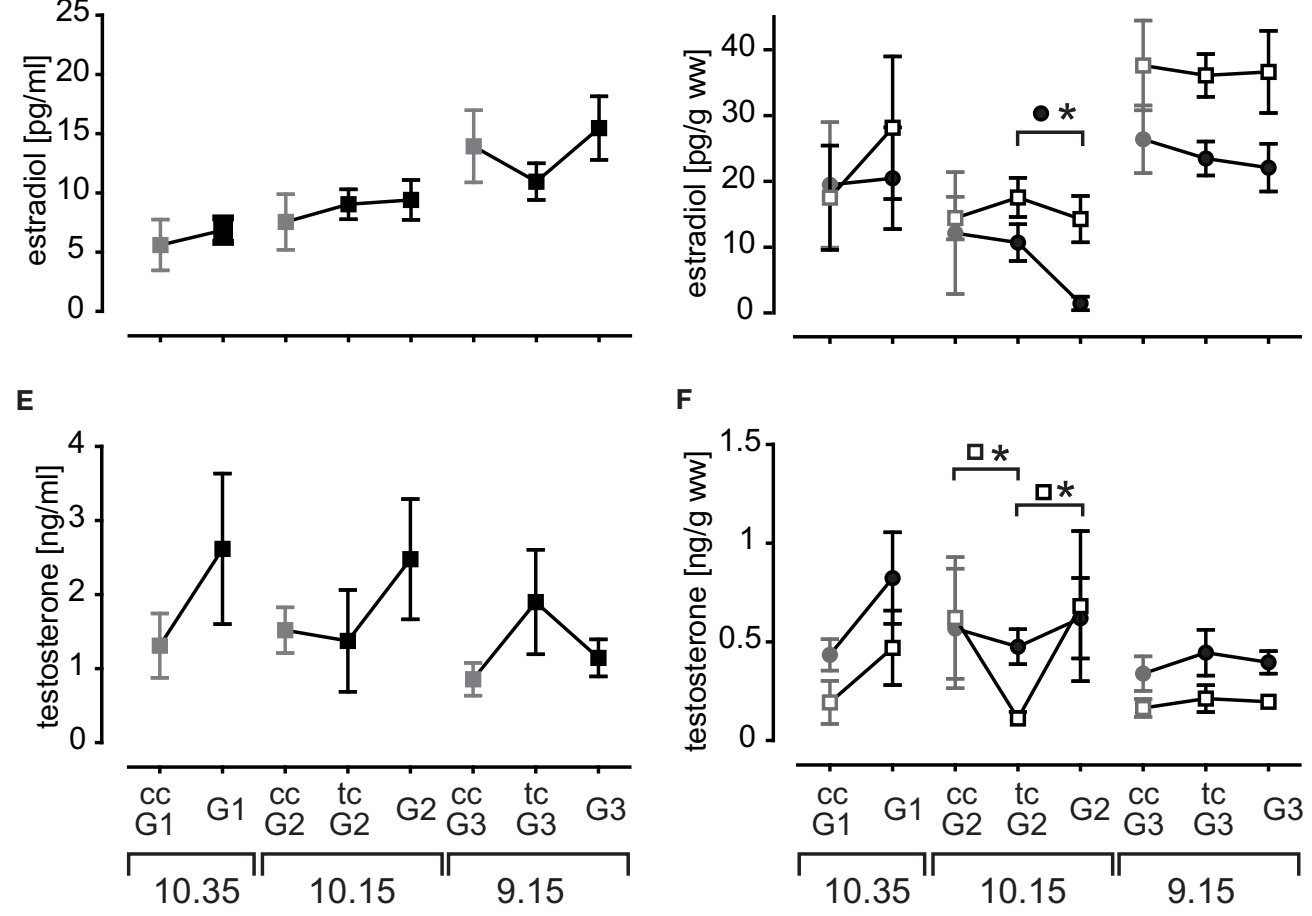

$\mathbf{F}$

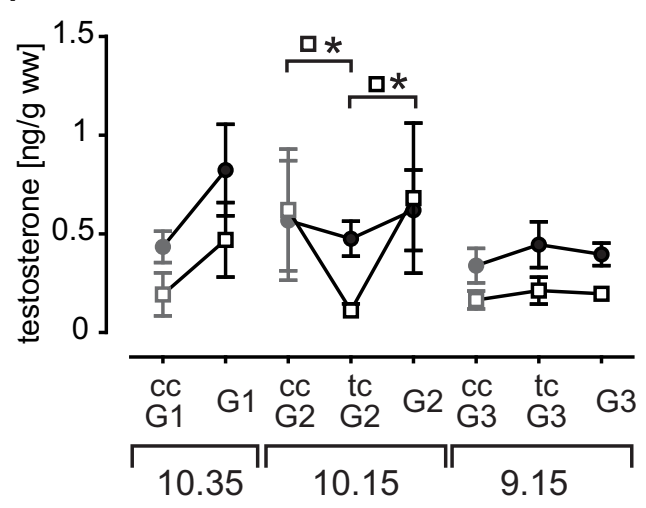

FIGURE 2 |The profile of endogenous hormone titers measured in serum (left panel) and brain tissue probes (right panel). Given are mean hormone concentrations for (A) corticosterone serum, (B) corticosterone brain tissue, (C) estradiol serum, (D) estradiol brain tissue, (E) testosterone serum, and (F) testosterone brain tissue probes. Time of sacrifice was dependent on session

sacrifice, control groups required careful consideration: To control for the systematic variation of the daytime of sacrifice between groups (due to different trial numbers in the three sessions), we compared hormone levels to chamber controls. Effects seen here could either be of acute nature, i.e., arising from the experience of the very session, or could be a delayed effect such as a shift of circadian release patterns due to a prior session. This scenario is plausible for several reasons: the training was performed during the animals' sleep cycle, food availability is known to be a strong modulator of circadian rhythmicity (Nelson et al., 1975), and calorie restriction is known to affect steroid hormone release patterns (Krieger, 1974; Itoh et al., 1980; Chacon et al., 2004). To disambiguate between these two scenarios we had a trained control group, which was sacrificed $24 \mathrm{~h}$ after their last training. Thus, late effects are indicated by a difference between chamber controls and trained controls. length and is indicated at the bottom. Chamber controls (untrained animals) are depicted in gray and abbreviated with ccG. Trained controls (tcG) were sacrificed $24 \mathrm{~h}$ after their last training at the same time point as the corresponding training group and controlled for long-term effects of the training on the hormone household.
Corticosterone in trained animals was generally slightly elevated as compared to chamber controls and chamber controls expressed successively increasing levels with earlier sacrifice time (Figure 2A). Serum levels were prominently elevated after session $3(p=0.005)$. Brain tissue probes expressed the same pattern as serum probes, but there was only a trend in session 3 (PFC $p=0.061$, hippocampus $p=0.075$; Figure 2B). Corticosterone in prefrontal probes was also significantly increased after session $2(p=0.01)$. The hippocampal concentrations of tcG2 were elevated $(p=0.014)$, pointing to a possible small shift of diurnal release pattern.

Estradiol serum levels expressed overall a similar time course as corticosterone serum, though no significant changes were observed (Figure 2C). More prominent is the brain tissue profile (Figure 2D) with two important aspects: (1) hippocampal and PFC probes do not behave synchronously as for example seen in corticosterone and 
(2) this becomes most evident in the acute drop of PFC estradiol after session $2(p=0.011)$. The magnitude of hormone concentration distribution for estradiol was also remarkable as hippocampal levels $(26.95 \mathrm{pg} / \mathrm{g} \mathrm{ww})$ were almost tripled compared to serum samples $(10.61 \mathrm{pg} / \mathrm{ml} ; p<0.001)$. These findings suggest local metabolism of estradiol in the investigated brain regions.

Testosterone levels expressed an enormous variability in trained rats (Figure 2E). In general, testosterone levels were rather acutely elevated after the first two sessions, and decreased compared to trained controls after the recall (session 3). Hippocampal testosterone in tcG2 was significantly decreased compared to chamber controls $(p=0.039)$ and acutely trained animals (G2 $p=0.046$; Figure $2 \mathbf{F}$ ). Thus hippocampal testosterone is acutely increased after session 2 .

\section{CORRELATION OF HORMONE TITERS AND BEHAVIORAL DATA: TESTOSTERONE WAS INTIMATELY LINKED TO THE RECALL SESSION}

A correlative analysis was performed to identify behavioral parameters related to individual hormone titers. There were multiple correlations for corticosterone and testosterone, but fewer for estradiol (Figure 3). A remarkable number of correlations of serum and PFC testosterone occurred in the last session (Figure 3A), while corticosterone was rather linked to the second session and the same, but to a smaller extent, is true for estradiol.

The behavioral parameters analyzed were clustered into three classes according to their relevancy for the task. (1) Reference memory (RM) related parameters included visiting or inspecting non-baited holes as well as crossings of the animal into areas surrounding baited holes (special cell entries, see also Figure 1B). (2) Working memory (WM) related activities were defined as re-visiting or re-inspecting baited holes and the total number of double inspected or double visited holes. (3) Agility related parameters included speed, the percentage of time spent moving, the total number of holes visited or inspected and the abidance at the border of the board or in the center of the arena. Regarding corticosterone, mainly levels in the PFC were linked to RM relevant variables, while hippocampal and serum corticosterone were intimately linked to agility related parameters (Figure 3B). A large number of positive correlations to task unspecific parameters suggests an activity supporting effect of endogenous corticosterone. Hippocampal estradiol was linked to WM performance while, serum levels rather related to parameters usually used to identify anxiety, e.g., time spent along the border of the arena versus in the center. Serum testosterone was correlated with all classes of parameters, however, strongest with task relevant parameters (WM and RM related). Compared to serum and PFC levels, hippocampal testosterone expressed fewer correlations which were, however, even more clustered on $\mathrm{RM}$ related parameters. These findings were further supported by multiple linear regression analysis (Figure 3C). For corticosterone the most informative predictors as evaluated by multiple linear regression were distributed over all of the three parameter classes. Testosterone was stronger - though not exclusively - linked to RM related parameters.

Interestingly, correlations did not co-occur in all tissues to the same degree (Figure 3D). In most cases, a correlation with a certain parameter was present in one tissue only. This is especially true for estradiol and is another piece of evidence for an independent regulation of

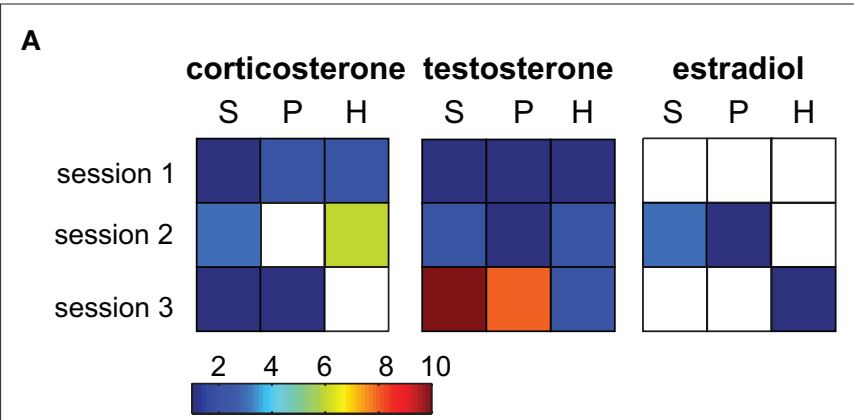

B

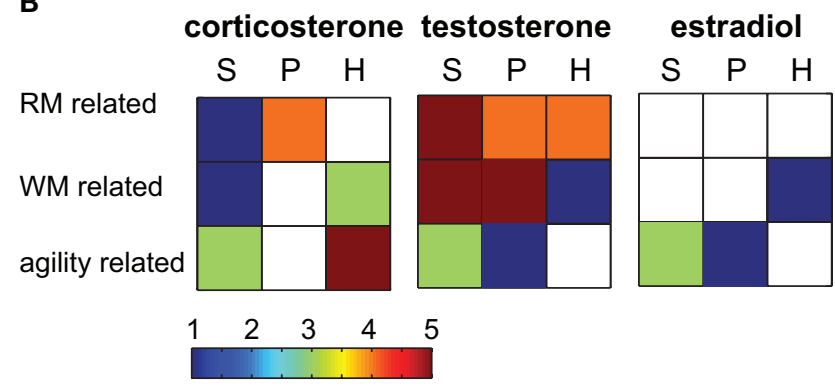

C

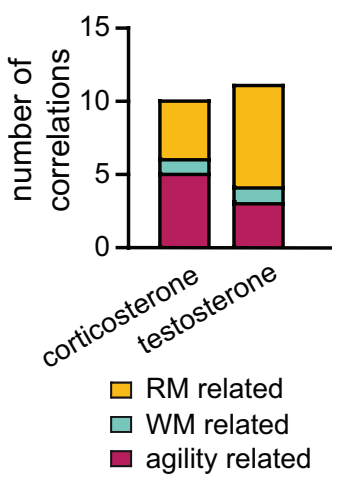

D

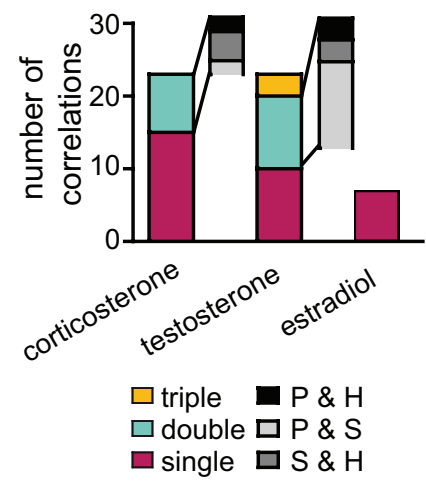

FIGURE 3 | Correlation analysis of hormones and behavioral data. To illustrate the relevancy of hormones over the training course, in (A) the number of significant correlations per session (acute groups G1, G2, and G3 only) are depicted color coded for each tissue type ( $\mathrm{S}=$ serum, $\mathrm{P}=\mathrm{PFC}$ and $\mathrm{H}=$ hippocampus). Testosterone expressed the highest number of significant correlations in the last session. In (B) the same data was arranged with parameters classified into three behavioral categories: working memory (WM), reference memory (RM) or unspecific activity (agility) related.

(C) Outcome of the multiple regression analysis grouped according to the behavioral classification. (D) Illustrates the number of correlations occurring between a behavioral parameter and tissue types: if the parameter correlated significantly with one tissue only, it is termed "single," "double" for two tissues and triple if correlation occurred between the parameter and all three samples. The gray insets illustrate the tissue pairs present in the double correlation bar.

estradiol in the different tissues investigated. For testosterone, there were clearly strong parallels in the PFC and serum correlations. The hippocampus probes were less coupled to other probes.

Thus, the general picture emerges, that corticosterone expressed multiple correlations distributed over all training groups and over all parameter categories and was such non-specifically coupled to 
several behavioral components. On the other hand, testosterone was stronger related to RM related parameters and specifically strongly linked to session 3, a session in which learning experience modulates synaptic plasticity (Uzakov et al., 2005). A closer look at correlations of testosterone in the final session confirmed that serum concentrations were correlated to the same parameters as PFClevels (Figure 4A). The behavioral parameters involved can be split into two groups: Parameters indicating explorative behavior, such as the total number of holes inspected and visited correlated with lower coefficients, while task relevant parameters, e.g., RME, special cell entries, and WME, correlated with higher coefficients. Note that correlations to RM related parameters reverse the direction over

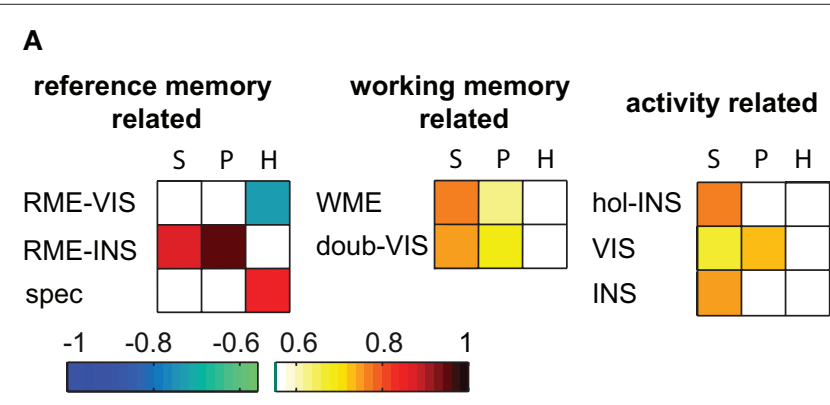

B
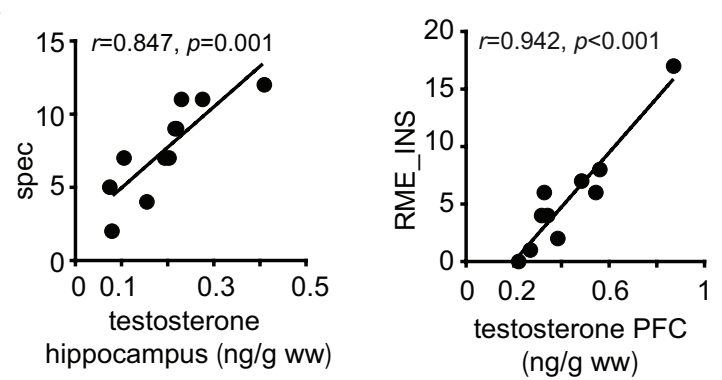

C

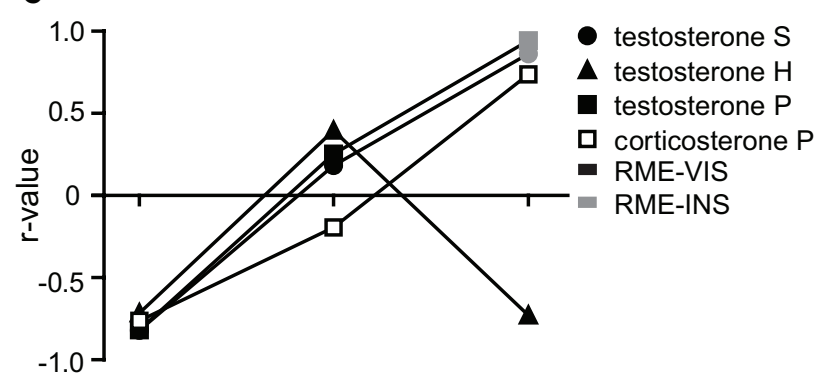

session $1 \quad$ session $2 \quad$ session 3

FIGURE 4 | Role of testosterone during recall. (A) The $r$-value of significant correlations of testosterone with behavior in session 3 are depicted color coded for each tissue type (S, serum; P, PFC; and H, hippocampus) and parameter. spec, special cell entries; INS, inspections, VIS, visits; doub-VIS, number of double visit holes; hol-INS, number holes inspected. (B) While hippocampal testosterone correlated with the task-promoting parameter special cell entries (spec) prefrontal testosterone correlated with RME-INS. (C) There was an inversion of correlation direction with RME-INS respective RME-VIS from early to late trials for PFC and serum testosterone as well as PFC corticosterone, but not for hippocampal testosterone. ( $\mathrm{S}=$ serum, $\mathrm{P}=\mathrm{PFC}$ and $\mathrm{H}=$ hippocampus). the course of the training (Figure 4C): while correlations with data from session 1 was negative (RME-VIS s1), the highly significant $(p<0.01)$ correlations of RME-INS, which are also the best predictors for PFC (Figure 4B) and serum, is positive. Intriguingly, this relation of prefrontal testosterone to task impairing parameters contrasts findings for hippocampal testosterone which correlated with task-promoting behavior, e.g., positively with special cell entries and negatively with RME-VIS (Figures 4A,B). Similar to prefrontal testosterone, also prefrontal corticosterone was highly linked to RME and expressed likewise a flip of correlation direction (Figure 4C). Another inversion was seen for corticosterone PFC and serum levels to abidance in the center (not shown). Thus, animals with high corticosterone levels in early sessions will spend less time in the center and commit less RME, but, when having high corticosterone levels in later trials, animals will spend more time in the center and commit more errors.

\section{PART 2}

In Part 1, we identified testosterone as the most relevant hormone regarding task relevant parameters in the final session. It was previously shown, that electrically induced early LTP in the dentate gyrus is reinforced by recall of the spatial memory into a late, protein synthesis-dependent form. In part 2 we therefore manipulated brain tissue testosterone levels and investigated the effects on behavior and LTP.

\section{Testosterone concentration at the time of LTP induction}

At the time of LTP induction, testosterone application induced higher testosterone levels only in the hippocampus $(p=0.024)$ but not in the PFC (Figure 5G). Levels were within the physiological range but may represent a ceiling concentration. The impact of vehicle application on corticosterone levels compared to unprepared controls may result from a handling effect, as the placement of the injector implies the removal of the animals from the cages (Figure 5H).

\section{Testosterone application in trained rats}

Testosterone impairs LTP reinforcement. Testosterone and vehicle substance was applied to the ventricle $30 \mathrm{~min}$ before LTP induction and thus $45 \mathrm{~min}$ before the animals were performing session 3 . Since establishment of a RM is crucial for cognitive reinforcement (Uzakov et al., 2005) only data from animals with a stable RM recall were included in electrophysiological analysis. Both potentials, the PSA and the fEPSP, stayed highly potentiated in controls $(n=6)$, while reinforcement was impaired in testosterone applied animals $(n=7)$, where signals returned to baseline values within $4 \mathrm{~h}$ (PSA) respective $5 \mathrm{~h}$ (fEPSP) after tetanus (Figures 5A,B). Testosterone treated animals and controls differed significantly in the protein synthesis-dependent phase (from 5 h onwards) in fEPSP $\left(F_{(1,11)}=6.514\right.$, $p=0.027)$ and were close to the significant criterion in the PSA values $\left(F_{(1,10)}=4.75, p=0.054\right)$. PSA values differed between groups at $24 \mathrm{~h}(p=0.020)$. There was no difference in the initial potentiation, measured shortly before behavioral manipulation.

Testosterone application disrupts coherency of behavior and electrophysiology. Investigated behavioral parameters did not differ between treated animals and controls. However, while in controls 
behavioral parameters correlated with electrophysiological data this coherency of behavior and electrophysiology was disrupted by testosterone treatment. In controls, the first PSA hour value after behavioral manipulation correlated significantly with latency $(r=-0.971, p=0.006)$ and the first fEPSP hour value with WMEINS $(r=0.929, p=0.007)$. This coherency was also previously described by Uzakov et al. (2005).

\section{Effects of testosterone application in untrained animals}

To identify whether effects of testosterone application on PSAand fEPSP-LTP are specific to training experience, we performed electrophysiological recordings in an untrained set of animals.

We first checked for effects on baseline transmission by using a $24 \mathrm{~h}$ recording protocol without LTP induction. Testosterone treated animals $(n=8)$ differed significantly in their PSA values from controls $\left(n=9 ; F_{(1,15)}=4.46, p=0.050\right)$ and the $24 \mathrm{~h}$ value was significantly lower $(p=0.028$; Figure $5 \mathrm{C})$. The fEPSP remained stable in testosterone treated animals $(n=7)$ as well as in controls $(n=8$; Figure 5D).

In the early LTP protocol (as used in trained animals), we found that groups differed significantly in fEPSP measures $\left(F_{(1,14)}=5.68\right.$, $p=0.032$; Figure 5F). While the fEPSP of control animals $(n=9)$ returned to baseline values within $5 \mathrm{~h}$, it remained highly potentiated throughout the $8 \mathrm{~h}$ recording in testosterone treated animals $(n=7)$. The PSA of controls $(n=8)$ and testosterone applied animals $(n=9)$ were similar in the induction phase and returned to baseline over the course of $5 \mathrm{~h}$ (testosterone) respective $7 \mathrm{~h}$ (control) after the weak tetanus (Figure 5E). Testosterone treated animals had a significantly lower $24 \mathrm{~h}$ PSA value than controls $(p=0.048)$.

To locate the site of action of testosterone, i.e., pre- versus postsynaptic, a paired pulse protocol was applied (Figure 5I). The protocol showed no significant differences between controls $(n=7)$ and testosterone applied animals $(n=7)$, arguing against the presynapse as effector site (data not shown).

\section{DISCUSSION}

The study aimed at investigating endogenous fluctuations of steroidal hormones throughout a spatial learning paradigm not only peripherally but also in task relevant brain regions. Our data supports the notion that metabolic processes occurring in local brain regions can modify steroid levels and we can further show that these effects are behaviorally relevant. After identifying testosterone as the hormone with the strongest link to task relevant parameters in the time period where synaptic plasticity in the dentate gyrus is modulated, the effects of steroid household manipulation was investigated in the second part of the study. Although the artificial rise of testosterone $45 \mathrm{~min}$ before the recall trial did not affect behavioral performance significantly, the effect on synaptic plasticity was pronounced. Testosterone application abolished LTP reinforcement.

\section{ENDOGENOUS HORMONES LINK TO BEHAVIOR Evidence for independent regulation}

Recent literature suggest that certain steroids like estradiol and testosterone can be produced by nervous tissue, which may allow brain region specific regulations of concentrations on a microdimensional scale (Agís-Balboa et al., 2006; Mukai et al., 2006; Ishii et al., 2007). In this study we dissected the brain regions hippocampus and PFC from animals undergoing a RM task containing WM elements. Estradiol levels in brain regions of these intact males expressed distinct and higher concentrations as compared to serum concentrations (cf. Barker and Galea, 2009), which is a prominent feature of neurosteroids (Corpéchot et al., 1981; Baulieu and Robel, 1998). In the case of testosterone, we could show at the individual level that tissue specific fluctuations are linked to learning task relevant behavior: While PFC concentrations of testosterone correlated mainly with the same parameters as serum testosterone, concentrations measured in the hippocampus, a region with neurosteroid production ability and rich aromatase activity, were linked in an opposite manner to RM performance in this final trial. Namely, hippocampal testosterone levels link to task-promoting parameters, while prefrontal testosterone correlated positively with parameters indicating poor memory formation. However, the hormone profile for this session did not show remarkable differences between the two brain regions. This is pointing out the importance of slight concentration modifications by local metabolism for brain region specific hormonal functions.

Other routes of local hormone level regulation may underlie the finding that brain concentrations of corticosterone remained unchanged in food-deprived animals, though the serum levels were significantly elevated. We suppose a higher clearance rate in the brain (Droste et al., 2008), possibly as a mechanism to maintain central functions of corticosterone independent from peripheral metabolic functions.

\section{Hormone fluctuations throughout the training: learning related or unrelated effects}

The prominent global increase in corticosterone during session 3 might be a matter of an unspecific rise due to the single day trial, as exposure of naïve animals to the holeboard induces a similar rise in serum corticosterone (Korz and Frey, 2004). Furthermore, corticosterone levels were only slightly elevated after repeated trials during sessions 1 and 2. However, we cannot rule out a task specific effect of corticosterone as well as of estradiol, which expresses a very similar profile. Both hormones have been reported to enhance longterm memory consolidation by post-training application (Packard and Teather, 1997; McGaugh and Roozendaal, 2002; Herbert et al., 2006; Fernandez et al., 2008). A similar role of endogenous levels and an interaction of these two hormones is feasible.

In contrast, testosterone levels, especially in the hippocampus, were elevated in session 2 as compared to their trained controls and lowered in session 3. During both sessions a high number of correlations of testosterone to behavioral performance could be observed, however very likely with a different functional background. Testosterone in session 3 shows tight coupling to RM performance. As animals usually acquire the task during session 2, the increase of testosterone levels is paralleled by learning related reward. In fact, androgens, and testosterone specifically, are known to have rewarding properties themselves (Packard et al., 1998), and have been shown to increase extracellular dopamine (de Souza Silva et al., 2009). Dopamine is involved in the consolidation of the RM (Korz and Frey, 2007). Thus the brain region specific modulation of testosterone provides a linkage and interface between catecholamines and hormones in the processing and consolidation of cognitive components of the task. 

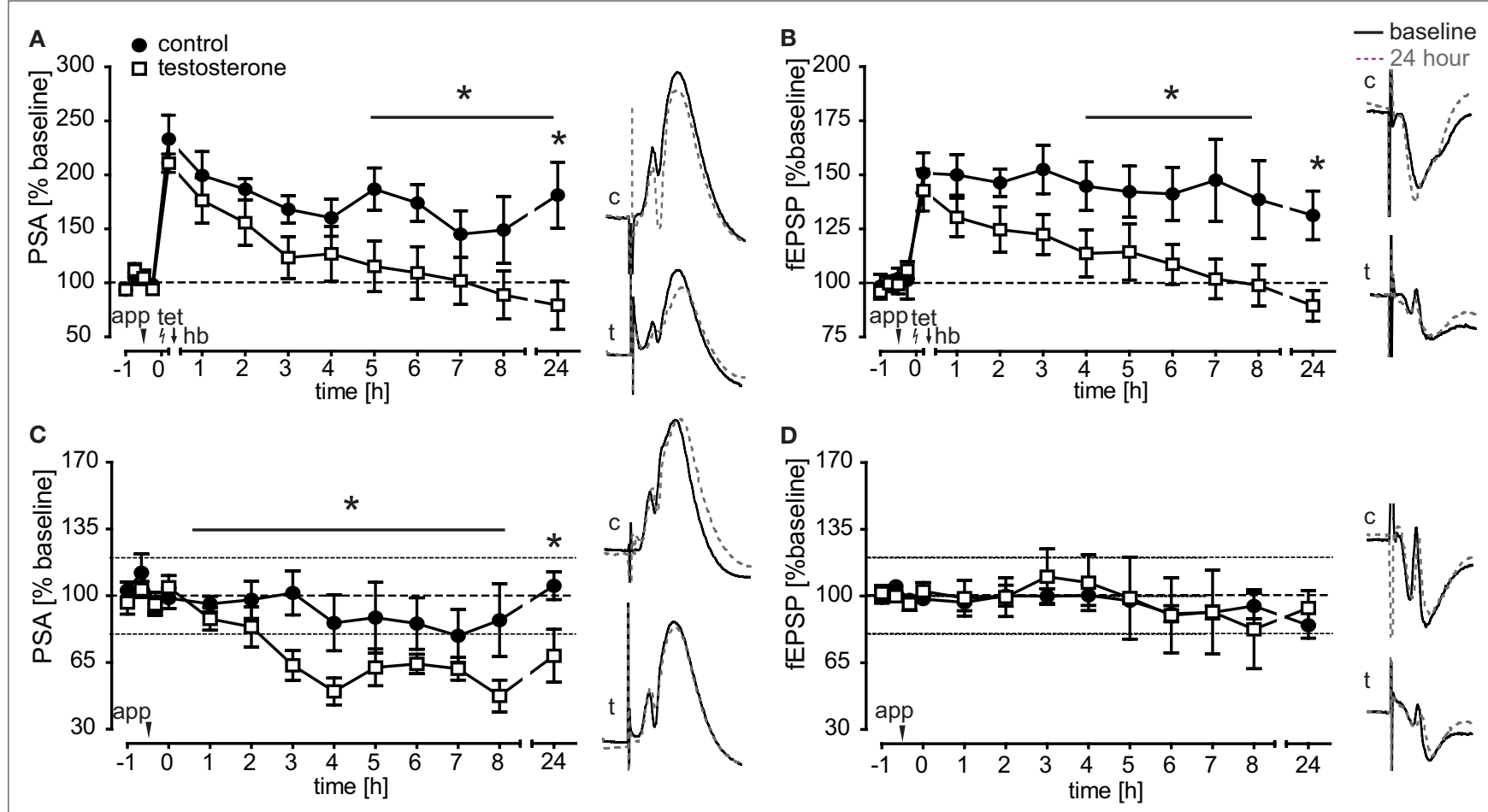

E
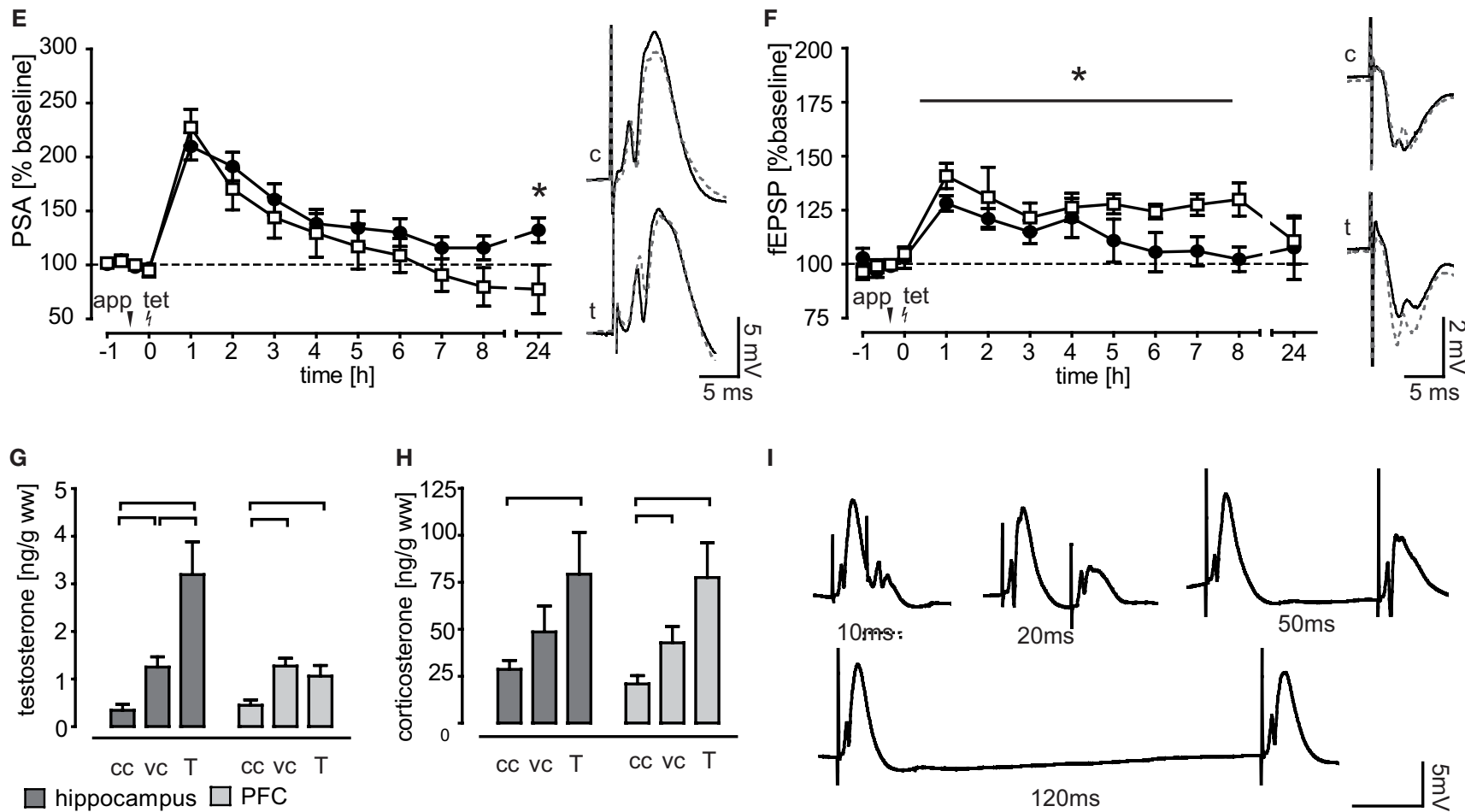

I

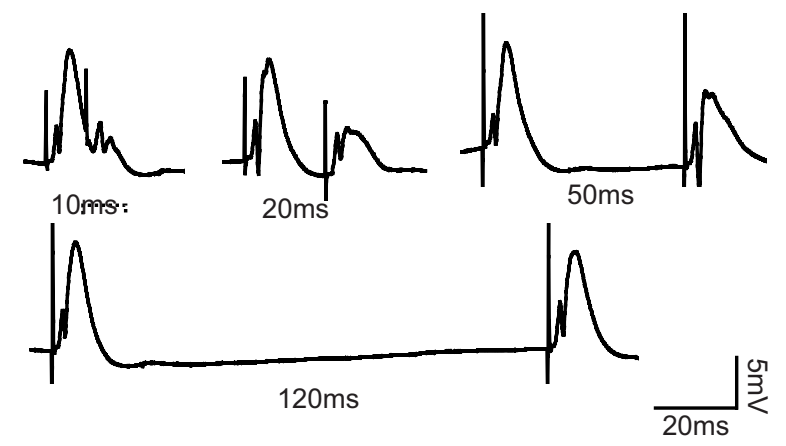

FIGURE 5 | Effect of testosterone application on field potential measurements. $(\mathbf{A}, \mathbf{B})$ Performance of a holeboard recall session (hb) after LTP induction (tet) led to LTP reinforcement in control animals for both, the PSA and the fEPSP. In contrast, intracerebroventricular application of testosterone (app) 30 min prior to LTP induction impaired the behavioral reinforcement of LTP. (C,D) In untrained animals, testosterone application reduced the PSA-baseline

transmission while leaving the fEPSP intact. (E,F) PSA values of testosterone treated, untrained animals undergoing an LTP induction protocol were reduced at $24 \mathrm{~h}$ after induction, while fEPSP potentiation was prolonged for $8 \mathrm{~h}$. $\mathbf{( G , H )}$ Brain tissue testosterone and corticosterone levels of chamber controls (cc, $n=16$ ), vehicle applied (vc, $n=9$ ), and animals receiving $1 \mu \mathrm{g}$ testosterone (T, $n=9$ ). (I) Example traces of PSA recordings in the paired pulse protocol. 


\section{Endogenous testosterone and searching strategy}

Over viewing the numerous behavioral parameters correlated to testosterone in late sessions, the role of endogenous testosterone may be best described as being related to a highly active, explorative behavior without a defined searching strategy: Many special cell crossings and WME indicate that animals with high testosterone are repeatedly searching in the baited area and suggest a relation to perseverance. This interpretation is in accordance with effects of long-term testosterone treatment on perseverance (van Hest et al., 1989). However, high testosterone does not lead to faster success in finding all pellets, as there were no correlations to latency. Rather the balance between levels in different regions (see above) and/or to other hormones may be crucial.

Both, testosterone and corticosterone, show an inversion of the sign of correlation with RME in the PFC over the training course. This may indicate a synergistic interaction between the hormones at the receptor or protein level. Furthermore, on the behavioral level it indicates a change of function over time from emotional to cognitive information processing. High corticosterone and testosterone levels in early training stages relate to rather passive/anxious behavior indicated by correlating negatively with RME - a parameter which at this stage rather reflects activity as a $\mathrm{RM}$ has not been formed yet - as well as by a negative correlation of corticosterone with time in the center. Conversely, in the last trial the direction reverses: high corticosterone correlates with more time in the center and concentrations of both hormones correlate with increased RME.

The positive correlations of endogenous testosterone with WME contrast studies which rather suggest a positive modulation of WM performance by testosterone (Daniel et al., 2003; Gibbs, 2005; Sandstrom et al., 2006; Alejandre-Gomez et al., 2007). We assume that this discrepancy arises from essentially different mechanisms of testosterone in physiological conditions versus conditions of depletion, e.g., in castrates.

Thus, our correlative analyses suggest a role of testosterone and metabolites in memory formation. The fact that the exogenous application of testosterone yielded no differences in behavioral performance may seem contradictive. However, the effects on synaptic plasticity occurred on a long time scale ( $5 \mathrm{~h}$ post application), suggesting that the time between application and behavioral testing might have been simply too short to observe a behavioral impact. Additionally, testosterone may be more effective to modulate learning related behavior in the acquisition phase. Naghdi et al. (2005) only report impairments by acute intrahippocampal testosterone application prior to training in early learning stages in the water maze, but not at the end of their training protocol. It is therefore likely that application earlier during the training protocol may influence memory formation.

\section{TESTOSTERONE APPLICATION AFFECTS SYNAPTIC AND NEURONAL PLASTICITY IN THE DENTATE GYRUS}

Manipulation of the hormone status by icv application of testosterone affected synaptic and neuronal plasticity differentially in trained versus untrained animals. Testosterone administration in untrained animals had depressing effects on the PSA-baseline and reinforced the fEPSP-LTP after a weak tetanus. These effects are likely not mediated by presynaptic mechanisms, as suggested by our paired pulse experiments, which is in line with a study by Sakata et al. (2000). The effect of testosterone in untrained animals can be summarized as a decrease in the EPSP-spike coupling in a time window from 3 to approximately $8 \mathrm{~h}$ post application. Such an effect can potentially be induced by modulation of GABAergic inhibition. The testosterone metabolite $3 \alpha$-diol is known to act in a benzodiazepine like fashion (Frye et al., 1996), so that increased inhibition could underlie the effects on the PSA in the baseline experiment. Conversely, effects on early LTP parallel the potent GABA $_{A}$ antagonist bicuculline, which induces higher fEPSP values without potentiation of the PSA (Tomasulo et al., 1991). This effect is potentially caused by estradiol, as multiple evidence suggests that estradiol reduces GABAergic inhibition (Weiland et al., 1997; Murphy et al., 1998; Kim et al., 2006; Ledoux et al., 2009). This explanation implies that the stimulation protocol affected metabolic conversion of testosterone. A stimulation protocol dependent conversion of testosterone to estradiol is indeed conceivable, since it is known that aromatase is differentially affected by activation of NMDA receptors (activated during the tetanus) and kainate and AMPA receptors (under basal conditions) (Hojo et al., 2004; Balthazart et al., 2006).

In trained animals testosterone impaired cognitive reinforcement significantly in both PSA- and fEPSP-LTP. The time course of PSA and fEPSP values is highly similar to effects of the $\beta$-adrenergic antagonist propranolol on cognitive reinforcement (Korz and Frey, 2007). Testosterone is known to diminish the amount of noradrenaline by actions on expression (Thanky et al., 2002) and activity of tyrosine hydroxylase, the rate limiting enzyme in noradrenaline production (Beattie and Martin, 1976; Engel et al., 1979). In contrast to a rapid and complete blockage of $\beta$-adrenergic receptors, testosterone is likely to only gradually decrease noradrenaline availability. This may explain the discrepancy to the effects of propranolol on behavior in the study by Korz and Frey (2007). Noradrenaline may additionally also be involved in the observed effects in untrained animals for LTP (Robinson and Racine, 1985) and baseline experiments (Pelletier et al., 1994). The identification of the cellular mechanisms involved in the bidirectional effects of testosterone on synaptic transmission is a challenge for future studies.

In which way do these effects on plasticity relate to memory? A possible scenario is the following: Testosterone application as such may act as a behaviorally relevant stimulus, e.g., as reward signal, which triggers an event related memory trace. This trace may, in the temporal relation to the weak tetanus, be consolidated in untrained animals, as reflected by the reinforcement of fEPSPLTP. A similar effect has been described by Seidenbecher et al. (1997) using aversive or appetitive stimuli for LTP reinforcement. In trained animals the event related memory trace could interfere with the spatial memory trace. Thus, both memory traces compete for plasticity-related proteins (cf. Fonseca et al., 2004) which may result in the observed impairment of LTP-reinforcement. In addition, testosterone application may also cause the interference with memory traces not necessarily related to the task relevant spatial memory, and may thereby cause a discrepancy between synaptic plasticity and behavior related to spatial learning. The complete lack of correlations of field potentials with spatial behavior in testosterone treated animals is pointing toward such a decoupling. 


\section{CONCLUSION}

It stands to reason that gonadal steroids and their different metabolites should be taken into consideration when memory formation and synaptic plasticity is under investigation in freely moving, intact animals. If solely relying on serum hormone levels, important details may be overlooked. Measurement of brain tissue concentrations of neurosteroids and related enzymes, on the other hand, may prove crucial to understand hormone-behavior relationships. This

\section{REFERENCES}

Agís-Balboa, R. C., Pinna, G., Zhubi, A., Maloku, E., Veldic, M., Costa, E., and Guidotti, A. (2006). Characterization of brain neurons that express enzymes mediating neurosteroid biosynthesis. Proc. Natl. Acad. Sci. U.S.A. 103, 14602-14607.

Alejandre-Gomez, M., Garcia-Segura, L. M., and Gonzalez-Burgos, I. (2007). Administration of an inhibitor of estrogen biosynthesis facilitates working memory acquisition in male rats. Neurosci. Res. 58, 272-277.

Balthazart, J., Baillien, M., and Ball, G. F. (2006). Rapid control of brain aromatase activity by glutamatergic inputs. Endocrinology 147, 359-366.

Barker, J. M., and Galea, L. A. (2009). Sex and regional differences in estradiol content in the prefrontal cortex, amygdala and hippocampus of adult male and female rats. Gen. Comp. Endocrinol. 164, 77-84.

Baulieu, E. E., and Robel, P. (1998). Dehydroepiandrosterone (DHEA) and dehydroepiandrosterone sulfate (DHEAS) as neuroactive neurosteroids. Proc. Natl. Acad. Sci. U.S.A. 95, 4089-4091.

Beattie, C. W., and Martin, M. M. (1976). Influence of castration and testosterone replacement on hypothalamic tyrosine hydroxylase activity in the rat. Experientia 32, 1076-1077.

Cato, A. C., Ponta, H., and Herrlich, P. (1992). Regulation of gene expression by steroid hormones. Prog. Nucleic Acid Res. Mol. Biol. 43, 1-36.

Chacon, F., Cano, P., Jimenez, V., Cardinali, D. P., Marcos, A., and Esquifino, A. I. (2004). 24-hour changes in circulating prolactin, follicle-stimulating hormone, luteinizing hormone, and testosterone in young male rats subjected to calorie restriction. Chronobiol. Int. 21, 393-404.

Corpéchot, C., Robel, P., Axelson, M., Sjövall, J., and Baulieu, E. E. (1981). Characterization and measurement of dehydroepiandrosterone sulfate in rat brain. Proc. Natl. Acad. Sci. U.S.A. 78, 4704-4707.

Daniel, J. M., Winsauer, P. J., and Moerschbaecher,J.M.(2003).Castration in rats impairs performance during acquisition of a working memory task and exacerbates deficits in working memory produced by scopolamine and mecamylamine. Psychopharmacology (Berl.) 170, 294-300.

de Souza Silva, M. A., Mattern, C., Topic, B., Buddenberg, T. E., and Huston, J. P. (2009). Dopaminergic and serotonergic activity in neostriatum and nucleus accumbens enhanced by intranasal administration of testosterone. Eur. Neuropsychopharmacol. 19, 53-63.

Diamond, M. I., Miner, J. N., Yoshinaga, S. K., and Yamamoto, K. R. (1990). Transcription factor interactions: selectors of positive or negative regulation from a single DNA element. Science 249, 1266-1272.

Droste, S. K., de Groote, L., Atkinson, H. C., Lightman, S. L., Reul, J. M. H. M., and Linthorst, A. C. E. (2008). Corticosterone levels in the brain show a distinct ultradian rhythm but a delayed response to forced swim stress. Endocrinology 149, 3244-3253.

Engel, J.,Ahlenius, S.,Almgren, O., Carlsson, A., Larsson, K., and Södersten, P. (1979). Effects of gonadectomy and hormone replacement on brain monoamine synthesis in male rats. Pharmacol. Biochem. Behav. 10, 149-154.

Fernandez, S. M., Lewis, M.C., Pechenino, A. S., Harburger, L. L., Orr, P. T., K. M. (2008). Estradiol-induced enhancement of object memory consolidation involves hippocampal extracellular signal-regulated kinase activation and membrane-bound estrogen receptors. J. Neurosci. 28, 8660-8667.

Fonseca, R., Nagerl, U. V., Morris, R. G., and Bonhoeffer, T. (2004). Competing for memory: hippocampal LTP under regimes of reduced protein synthesis. Neuron 44, 1011-1020.

Frye, C. A., Keuren, K. R. V., and Erskine, M. S. (1996). Behavioral effects of 3 alpha-androstanediol. I: modulation of sexual receptivity and promotion of GABA-stimulated chloride flux. Behav. Brain Res. 79, 109-118.

Gibbs, R. B. (2005). Testosterone and estradiol produce different effects on cognitive performance in male rats. Horm. Behav. 48, 268-277.

Hajszan, T., MacLusky, N. J., Johansen, J. A., Jordan, C. L., and Leranth, C. Gresack, J. E., Schafe, G. E., and Frick,

study provides first evidence for a functional link of local steroid production to behavior.

\section{ACKNOWLEDGMENTS}

This study was supported by grants from the German Research Council (DFG-Ko-1540/5-1), the Center of Behavioral Brain Sciences, Magdeburg and European funding from EFRE (ZVOH TP5/1) to Volker Korz.

(2007). Effects of androgens and estradiol on spine synapse formation in the prefrontal cortex of normal and testicular feminization mutant male rats. Endocrinology 148, 1963-1967.

Hajszan, T., MacLusky, N. J., and Leranth, C. (2008). Role of androgens and the androgen receptor in remodeling of spine synapses in limbic brain areas. Horm. Behav. 53, 638-646.

Handa, R. J., Hejna, G. M., and Lorens, S. A. (1997). Androgen inhibits neurotransmitter turnover in the medial prefrontal cortex of the rat following exposure to a novel environment. Brain Res. 751, 131-138.

Herbert, J., Goodyer, I. M., Grossman, A. B., Hastings, M. H., de Kloet, E. R., Lightman, S. L., Lupien, S. J., Roozendaal, B., and Seckl, J. R. (2006). Do corticosteroids damage the brain? J. Neuroendocrinol. 18, 393-411.

Herman,J.P.(1993). Regulation of adrenocorticosteroid receptor mRNA expression in the central nervous system. Cell. Mol. Neurobiol. 13, 349-372.

Hojo, Y., Hattori, T.-A., Enami, T. Furukawa, A., Suzuki, K., Ishii, H.-T., Mukai, H., Morrison, J. H., Janssen, W. G. M., Kominami, S., Harada, N Kimoto, T., and Kawato, S. (2004). Adult male rat hippocampus synthesizes estradiol from pregnenolone by cytochromes P45017alpha and P450 aromatase localized in neurons. Proc Natl. Acad. Sci. U.S.A. 101, 865-870.

Ishii, H., Tsurugizawa, T., Ogiue-Ikeda, M. Asashima, M., Mukai, H., Murakami, G., Hojo, Y., Kimoto, T., and Kawato, S. (2007). Local production of sex hormones and their modulation of hippocampal synaptic plasticity. Neuroscientist 13, 323-334.

Itoh, S., Katsuura, G., and Hirota, R. (1980). Conditioned circadian rhythm of plasma corticosterone in the rat induced by food restriction. Jpn. J. Physiol. 30, 365-375.

Joels, M., and de Kloet, E. R. (1989). Effects of glucocorticoids and norepinephrine on the excitability in the hippocampus. Science 245, 1502-1505.

Kim, M. T., Soussou, W., Gholmieh, G., Ahuja, A., Tanguay, A., Berger, T. W., and Brinton, R. D. (2006). 17betaestradiol potentiates field excitatory postsynaptic potentials within each subfield of the hippocampus with greatest potentiation of the associational/commissural afferents of CA3. Neuroscience 141, 391-406.

Korz, V., and Frey, J. U. (2004). Emotional and cognitive reinforcement of rat hippocampal long-term potentiation by different learning paradigms. Neuron Glia Biol. 1, 253-261.

Korz, V., and Frey, J. U. (2007). Hormonal and monoamine signaling during reinforcement of hippocampal long-term potentiation and memory retrieval. Learn. Mem. 14, 160-166.

Krieger, D. T. (1974). Food and water restriction shifts corticosterone, temperature, activity and brain amine periodicity. Endocrinology 95, 1195-1201.

Ledoux, V. A., Smejkalova, T., May, R. M., Cooke, B. M., and Woolley, C. S. (2009). Estradiol facilitates the release of neuropeptide $\mathrm{Y}$ to suppress hippocampus-dependent seizures. $J$ Neurosci. 29, 1457-1468.

MacLusky, N. J., Walters, M. J., Clark, A. S., and Toran-Allerand, C. D. (1994). Aromatase in the cerebral cortex, hippocampus, and mid-brain: ontogeny and developmental implications. Mol. Cell. Neurosci. 5, 691-698.

McEwen, B. S., Gerlach, J. L., Luine, V. N., and Lieberburg, I. (1977). Neural steroid hormone receptors. Psychoneuroendocrinology 2, 249-255.

McGaugh, J. L., Gold, P. E., Van Buskirk, R., and Haycock, J. (1975). Modulating influences of hormones and catecholamines on memory storage processes. Prog. Brain Res. 42, 151-162.

McGaugh, J. L., and Roozendaal, B. (2002). Role of adrenal stress hormones in forming lasting memories in the brain. Curr. Opin. Neurobiol. 12, 205-210.

Mukai, H., Takata, N., Ishii, H.-T., Tanabe, N., Hojo, Y., Furukawa, A., Kimoto, T., and Kawato, S. (2006). Hippocampal synthesis of estrogens and androgens which are paracrine modulators of synaptic plasticity: synaptocrinology. Neuroscience 138, 757-764.

Murphy, D. D., Cole, N. B., Greenberger, V., and Segal, M. (1998). Estradiol increases dendritic spine density by reducing GABA neurotransmission 
in hippocampal neurons. J. Neurosci. 18, 2550-2559.

Naghdi, N., Majlessi, N., and Bozorgmehr, T. (2005). The effect of intrahippocampal injection of testosterone enanthate (an androgen receptor agonist) and anisomycin (protein synthesis inhibitor) on spatial learning and memory in adult, male rats. Behav. Brain Res. 156, 263-268.

Nelson, W., Scheving, L., and Halberg, F. (1975). Circadian rhythms in mice fed a single daily meal at different stages of lighting regimen. J. Nutr. 105, 171-184.

Packard, M. G., Schroeder, J. P., and Alexander, G. M. (1998). Expression of testosterone conditioned place preference is blocked by peripheral or intra-accumbens injection of alpha-flupenthixol. Horm. Behav. 34, 39-47.

Packard, M. G., and Teather, L. A. (1997). Intra-hippocampal estradiol infusion enhances memory in ovariectomized rats. Neuroreport 8, 3009-3013.

Pavlides, C., Watanabe, Y., and McEwen, B. S. (1993). Effects of glucocorticoids on hippocampal long-term potentiation. Hippocampus 3, 183-192.
Paxinos, G., and Watson, C. (1998). The Rat Brain in Stereotaxic Coordinates, 2nd Edn. San Diego: Academic Press. Pelletier, M. R., Kirkby, R. D., Jones, S. J., and Corcoran, M. E. (1994). Pathway specificity of noradrenergic plasticity in the dentate gyrus. Hippocampus 4, 181-188.

Robinson, G. B., and Racine, R. J. (1985). Long-term potentiation in the dentate gyrus: effects of noradrenaline depletion in the awake rat. Brain Res. 325, 71-78.

Sakata, K., Tokue, A., and Kawai, N. (2000). Altered synaptic transmission in the hippocampus of the castrated male mouse is reversed by testosterone replacement. J. Urol. 163, 1333-1338.

Sandstrom, N. J., Kim, J. H., and Wasserman, M.A. (2006). Testosterone modulates performance on a spatial working memory task in male rats. Horm. Behav. 50, 18-26.

Seidenbecher, T., Reymann, K. G., and Balschun, D. (1997). A post-tetanic time window for the reinforcement of long-term potentiation by appetitive and aversive stimuli. Proc. Natl. Acad. Sci. U.S.A. 94, 1494-1499.

Sinopoli, K. J., Floresco, S. B., and Galea, L. A. (2006). Systemic and local administration of estradiol into the prefrontal cortex or hippocampus differentially alters working memory. Neurobiol. Learn. Mem. 86, 293-304.

Thanky, N.R.,Son, J.H., and Herbison,A.E. (2002).Sex differences in the regulation of tyrosine hydroxylase gene transcription by estrogen in the locus coeruleus of TH9-LacZ transgenic mice. Brain Res. Mol. Brain Res. 104, 220-226.

Tomasulo, R. A., Levy, W. B., and Steward, O. (1991). LTP-associated EPSP/spike dissociation in the dentate gyrus: GABAergic and non-GABAergic components. Brain Res. 561, 27-34.

Uzakov, S., Frey, J. U., and Korz, V. (2005). Reinforcement of rat hippocampal LTP by holeboard training. Learn Mem. 12, 165-171.

van Hest, A., van Haaren, F., and van de Poll, N. E. (1989). Perseverative responding in male and female Wistar rats: effects of gonadal hormones. Horm. Behav. 23, 57-67.

Wehrenberg, U., Prange-Kiel, J., and Rune, G. M. (2001). Steroidogenic factor-1 expression in marmoset and rat hippocampus: co-localization with StAR and aromatase. J. Neurochem. 76 , 1879-1886.
Weiland, N. G., Orikasa, C., Hayashi, S., and McEwen, B.S. (1997). Distribution and hormone regulation of estrogen receptor immunoreactive cells in the hippocampus of male and female rats. J. Comp. Neurol. 388, 603-612.

Conflict of Interest Statement: The authors declare that the research was conducted in the absence of any commercial or financial relationships that could be construed as a potential conflict of interest.

Received: 17 August 2010; accepted: 01 December 2010; published online: 17 December 2010.

Citation: Schulz K and Korz V (2010) Hippocampal testosterone relates to reference memory performance and synaptic plasticity in male rats. Front. Behav. Neurosci. 4:187. doi: 10.3389/ fnbeh.2010.00187

Copyright $(\odot 2010$ Schulz and Korz. This is an open-access article subject to an exclusive license agreement between the authors and the Frontiers Research Foundation, which permits unrestricted use, distribution, and reproduction in any medium, provided the original authors and source are credited. 